Itinéraires Itinéraires

Littérature, textes, cultures

2015-2 | 2016

Stumbling blocks. Entraves et obstacles aux circulations

\title{
Nouvelles perspectives sur l'accentuation des emprunts en anglais contemporain
}

New Perspectives on the Stressing of Loanwords in English

\section{Pierre Fournier}

\section{(2) OpenEdition}

Journals

Édition électronique

URL : http://journals.openedition.org/itineraires/2827

DOI : $10.4000 /$ itineraires.2827

ISSN : 2427-920X

Éditeur

Pléiade

Référence électronique

Pierre Fournier, « Nouvelles perspectives sur l'accentuation des emprunts en anglais contemporain », Itinéraires [En ligne], 2015-2 | 2016, mis en ligne le 15 février 2016, consulté le 06 mai 2019. URL:

http://journals.openedition.org/itineraires/2827 ; DOI : 10.4000/itineraires.2827

Ce document a été généré automatiquement le 6 mai 2019.

\section{c) $($ ) $(9)$}

Itinéraires est mis à disposition selon les termes de la licence Creative Commons Attribution - Pas

d'Utilisation Commerciale - Pas de Modification 4.0 International. 


\title{
Nouvelles perspectives sur l'accentuation des emprunts en anglais contemporain
}

\author{
New Perspectives on the Stressing of Loanwords in English
}

\author{
Pierre Fournier
}

\section{Introduction}

1 L'accentuation, et plus particulièrement l'assignation de l'accent principal, des emprunts en anglais contemporain bénéficient d'un traitement équivalent au reste du lexique dans les ouvrages de référence consacrés au sujet. Le paramètre déterminant est celui du poids syllabique selon lequel les syllabes lourdes attirent l'accent. Pour autant, si le poids syllabique joue un rôle certes non négligeable dans l'accentuation des emprunts en anglais ${ }^{1}$, d'autres facteurs relevant d'une logique totalement différente semblent supplanter le paramètre du poids syllabique. Ces derniers sont liés au degré de transfert des données phonologiques de la langue source à la langue réceptrice lors du phénomène d'emprunt. En effet, selon la langue à laquelle l'anglais a emprunté, le taux de conservation de la syllabe accentuée du terme d'origine est plus ou moins fort. L'emprunt peut ainsi conserver la position de la syllabe accentuée de la langue source ou au contraire, la reproduction de la syllabe accentuée du terme dans sa langue d'origine est entravée et une réinterprétation de l'accentuation de l'emprunt basée sur des critères propres à l'anglais intervient. Cette étude s'inscrit dans le courant théorique de la Loan Phonology, créé dans les années 1990 et qui étudie les phénomènes d'ordre phonologique lors des contacts entre langues. Des recherches sur l'assignation des accents lexicaux dans les emprunts ont déjà été menées (voir Svensson 2001 ou encore Kenstowicz et Sohn 2001). Deux scénarios sont généralement privilégiés : la structure accentuelle de la langue source est conservée ou au contraire, il y a adaptation au système accentuel de la langue réceptrice. Le présent article est centré sur l'étude de l'accentuation des emprunts italiens, japonais et français en anglais contemporain. Le choix des trois langues est tout 
sauf un hasard puisque l'anglais a, d'une part, beaucoup emprunté à ces trois langues au cours de son histoire, permettant ainsi un traitement quantitatif de la question, mais d'autre part, car un phénomène distinct à chacune des langues et directement lié à l'étymologie de l'emprunt est mis en évidence. Enfin, la présence, dans chacune de ces quatre langues, d'une syllabe accentuée, permet de procéder à un travail de comparaison. Trois langues sources, pour trois phénomènes distincts qui débouchent sur une nouvelle façon d'envisager les travaux sur l'accentuation des emprunts en anglais.

\section{L'accentuation des emprunts italiens en anglais}

\section{Critères de constitution du corpus}

2 La constitution d'un corpus représentatif d'emprunts en anglais obéit à l'application de critères stricts. L'étude étant centrée sur l'accentuation, deux dictionnaires de référence sur la prononciation de l'anglais servent de bases de données, à savoir le Longman Pronunciation Dictionary (LPD) et le Cambridge English Pronouncing Dictionary (EPD). Un premier corpus d'emprunts italiens en anglais élaboré à partir de l'EPD et du LPD (Fournier à paraître a) s'est enrichi des données de l'Oxford English Dictionary (OED) dans une étude ultérieure (Fournier à paraitre b). L'OED dans sa version en ligne (Simpson et Weiner 1989) constitue le stock lexical le plus important de la langue anglaise, et l'accès aux données étymologiques des emprunts s'avère fondamental dans la description de ces items.

3 Les corpus d'emprunts aux trois langues sont élaborés sur des bases similaires. Les termes obsolètes sont écartés car non seulement ils ne sont pas représentatifs de l'anglais contemporain, mais aussi car la base de données de l'OED n'attribue pas de transcription phonétique permettant de localiser la syllabe accentuée dans les termes obsolètes. De plus, la présence d'une transcription phonétique n'étant pas systématique dans l'OED, les emprunts sans transcription ne sont pas conservés. Seule la position de l'accent primaire est traitée dans cet article. Les variantes accentuelles présentes dans les bases de données sont conservées et seuls les emprunts directs figurent dans les corpus. En effet, un emprunt italien, japonais ou français ayant transité par une langue intermédiaire avant d'être emprunté par l'anglais pourrait avoir adopté des caractéristiques phonologiques de la langue intermédiaire, pouvant ainsi fausser les résultats concernant l'influence potentielle de la langue source sur la langue réceptrice. En cas de doute, l'emprunt n'est pas conservé, de même que si la présence d'un emprunt simultané est relevée. L'OED permet d'avoir des données relativement fiables concernant l'étymologie de chacun de ces termes. Enfin, la variété d'anglais américain est incluse dans l'analyse si elle s'avère potentiellement déterminante sur la position de l'accent primaire. Dans le cas contraire, c'est-à-dire si la variété d'anglais ne constitue pas un facteur déterminant dans l'accentuation des emprunts, la variété d'anglais sélectionnée est celle de l'anglais britannique.

4 Le corpus des emprunts italiens en anglais est donc élaboré à partir des trois sources dictionnairiques (LPD, EPD et OED). L'ouvrage de Laura Pinnavaia (2001) consacré aux emprunts italiens dans l'oxford English Dictionary s'avère d'une aide précieuse. Sont conservés des inventaires de Laura Pinnavaia, tous les emprunts au minimum trisyllabiques ${ }^{2}$ avec une structure en $\langle\mathrm{VCV}\rangle^{3}$. Après avoir vérifié dans l'OED que les emprunts retenus soient directs et que les structures syllabiques ${ }^{4}$ des deux langues 
présentent suffisamment de similitudes pour permettre une comparaison accentuelle, les schémas accentuels sont relevés dans l'OED, le LPD et l'EPD. Le corpus d'emprunts italiens en anglais s'élève ainsi à 408 items.

L'italien est une langue à accent lexical. Le travail de comparaison se trouve ainsi grandement facilité. La répartition des schémas accentuels en italien est la suivante : $80 \%$ des termes sont accentués sur la syllabe pénultième (en /(-)10/) contre $16 \%$ sur la syllabe antépénultième (en $/(-) 100 /)$ et $4 \%$ sur la finale (en $/-1 /)^{5}$. Afin de comparer les schémas accentuels des termes italiens avec leurs homologues empruntés en anglais, deux sources notant les syllabes accentuées en italien sont choisies: le dictionnaire unilingue de Francesco Sabatini et Vittorio Coletti (2009) et le dictionnaire français-italien de Paul Robert et d'Augusto Arizzi (1999). Néanmoins, sur les 408 emprunts directs répertoriés en anglais, 48 ne sont pas attestés dans les dictionnaires italiens. De plus, 48 cas de syllabes accentuées avec synérèse ${ }^{6}$ sont à noter dans les dictionnaires italiens, ainsi que 14 emprunts pour lesquels la détermination du nombre de syllabes varie en anglais et en italien. Le corpus d'analyse comprend donc 299 emprunts exploitables. Concernant les 299 items italiens, 236 sont accentués en /(-)10/ (78,9\%), 56 en /(-)100/ (18,7\%), 4 en /-1/ $(1,3 \%)$ et 3 attestent d'un phénomène de variation $(1,1 \%)$, ce qui correspond aux chiffres énoncés précédemment.

6 La prochaine sous-partie décrit les résultats obtenus en comparant l'accentuation des emprunts en anglais avec l'accentuation de ces mêmes items en italien.

\section{Résultats}

7 Le tableau 1 répertorie les cas de correspondance accentuelle ${ }^{7}$ totale entre l'anglais et l'italien :

Tableau 1. Correspondance accentuelle totale entre l'italien et les emprunts correspondants en anglais

\begin{tabular}{|c|c|c|}
\hline $\begin{array}{c}\text { correspondance totale } \\
/-\mathbf{1} / \rightarrow /-\mathbf{1} /{ }^{8}\end{array}$ & $\begin{array}{c}\text { correspondance totale } \\
/(-) \mathbf{1 0} / \rightarrow /(-) \mathbf{1 0} /\end{array}$ & $\begin{array}{c}\text { correspondance totale } \\
/(-) \mathbf{1 0 0} / \rightarrow /(-) \mathbf{1 0 0} /\end{array}$ \\
\hline $3 \mathrm{cas}$ & $233 \mathrm{cas}$ & $50 \mathrm{cas}$ \\
\hline exemples & exemples & exemples \\
\hline $\begin{array}{c}\text { oi'me } \rightarrow \text { oi'me } \\
\text { romani'ta } \rightarrow \text { Romani'tà } \\
\text { terribili'tà } \rightarrow \text { terribili'tà }\end{array}$ & $\begin{array}{c}\text { affetu'oso } \rightarrow \text { affetu'oso } \\
\text { do'gana } \rightarrow \text { do'gana } \\
\text { fu'rore } \rightarrow \text { fu'rore } \\
\text { ri'presa } \rightarrow \text { ri'presa }\end{array}$ & $\begin{array}{c}\text { al'tissimo } \rightarrow \text { al'tissimo } \\
\text { 'broccolo } \rightarrow \text { 'broccoli' } \\
\text { e'nergico } \rightarrow \text { e'nergico } \\
\text { 'timpano } \rightarrow \text { 'timpani }\end{array}$ \\
\hline
\end{tabular}

8 À ces 286 cas de correspondance totale, s'ajoutent 13 cas pour lesquels une correspondance accentuelle peut également être observée: 'agio $\rightarrow$ 'agio, 'aria $\rightarrow$ 'aria , au'relia $\rightarrow$ au'relia, impre'sario $\rightarrow$ impre'sario, 'oboe $\rightarrow$ 'oboe, ora'torio $\rightarrow$ ora'torio, 'palio $\rightarrow$ 'palio , pre'ludio $\rightarrow$ pre'ludio, pre'sepio $\rightarrow$ pre'sepio , ro'solio $\rightarrow$ ro'solio , sce'nario $\rightarrow$ sce'nario , si'nopia $\rightarrow$ si'nopia et 'studio $\rightarrow$ 'studio ${ }^{10}$. 
Quatre cas seulement ne montrent aucune correspondance accentuelle: bal'cone $\rightarrow$ 'balcony ${ }^{11}$, 'bucchero $\rightarrow$ buc'chero, cra'zia $\rightarrow$ 'crazia et 'porfido $\rightarrow$ por'fido. Enfin, 9 exemples de variation accentuelle sont avérés et répertoriés dans le tableau 2. La correspondance accentuelle n'est donc que partielle.

Tableau 2. Cas de correspondance accentuelle partielle entre l'italien et les emprunts correspondants en anglais

\begin{tabular}{|c|c|}
\hline italien & anglais \\
\hline 'bravo & 'bravo $(3)^{12}+/ 01 /$ EPD \\
\hline ca'sino / casi'no & ca'sino (3) \\
\hline in'cognita & in'cognita OED/EPD + /-10/ EPD \\
\hline in'cognito & in'cognito (3) + /-10/ (3) \\
\hline 'pergola & 'pergola (3) + /010/ LPD \\
\hline pie'ta & pie'ta (3) + 'pieta LPD/EPD \\
\hline 'rapido & 'rapido OED + /010/ OED \\
\hline ricer'care & ricer'care OED + /1000/ OED \\
\hline so'ave & so'ave $\mathrm{OED}+$ 'soave $\mathrm{LPD}^{13}$ \\
\hline
\end{tabular}

\section{Les emprunts à l'italien, un exemple parfait de conservation accentuelle}

Les données dictionnairiques démontrent un fort pourcentage de conservation de la position de l'accent lexical de l'italien lors du phénomène de transfert vers l'anglais. Sur 313 emprunts, 299 conservent l'accentuation du terme dans sa langue d'origine (soit 95,5\%), 9 cas correspondent partiellement par l'intermédiaire des variantes (soit 2,9\%) et seulement 4 cas de non correspondance accentuelle sont répertoriés (soit 1,6\%). La correspondance accentuelle est donc quasi systématique pour les emprunts à l'italien dans les structures en <-VCV\#> dans les sources dictionnairiques. L'emprunt lexical s'accompagne d'une conservation du schéma accentuel de la langue source. La majorité des items italiens est accentuée en /(-)10/ (environ $80 \%$ ). Pour autant, même les items accentués en /(-)100/ en italien conservent une accentuation sur la syllabe antépénultième en anglais lors du phénomène d'emprunt. Beaucoup de termes empruntés en anglais étant accentués sur la syllabe pénultième, un éventuel concours de circonstances peut être envisagé. Mais les correspondances accentuelles au niveau de la syllabe antépénultième entre l'italien et l'anglais démontrent que ce phénomène de correspondance est tout sauf accidentel. L'accentuation des emprunts italiens en anglais, de par le taux de correspondance quasi parfait, constitue un exemple remarquable de transmission de données phonologiques d'une langue à une autre. Les réflexions sur les raisons d'une telle correspondance demeurent encore à l'état embryonnaire, mais il 
semblerait que le canal d'intégration de ces emprunts à la langue anglaise soit une des pistes privilégiées. Comment les emprunts italiens ont-ils intégré la langue anglaise ? Par l'intermédiaire de l'écrit ou de l'oral ? La piste de l'oral semble la plus plausible, et ce, par l'intermédiaire de locuteurs bilingues (italien-anglais), d'où ce taux de correspondance élevé. L'étude des emprunts au japonais et au français démontre une tout autre réalité.

\section{L'accentuation des emprunts japonais en anglais}

\section{Critères de constitution du corpus et spécificités de l'accentuation en japonais}

11 Le corpus des emprunts japonais en anglais est élaboré à partir de l'outil de recherche avancée de l'OED grâce auquel il est possible de sélectionner les emprunts au japonais en anglais. Le nettoyage des données obtenues permet d'écarter les items ne provenant pas réellement du japonais car ayant transité par une langue intermédiaire, mais également les emprunts obsolètes, les traductions littérales ${ }^{14}$ du japonais et les emprunts « hybrides 15 ». Les mots complexes formés à partir d'une base japonaise et d'un affixe anglais sont également retirés du corpus car la position de l'accent primaire dans ces items est déterminée non pas par une potentielle influence de l'accentuation japonaise, mais au contraire par la présence de suffixes anglais contraignants (exemples : ibotenic, Nipponese). Enfin, les emprunts monosyllabiques ${ }^{16}$ ne sont pas conservés car ils ne permettent pas de comparaison accentuelle. Le corpus exploitable s'élève ainsi à 464 emprunts. La position de la syllabe accentuée est relevée dans l'OED, le LPD et l'EPD. Cent dix-sept emprunts japonais attestés dans l'OED figurent également dans l'EPD et/ou le LPD.

La comparaison accentuelle entre l'anglais et le japonais est complexe à cause des spécificités de l'accentuation japonaise. En effet, l'anglais est une langue à accent lexical ( stress-accent language) tandis que le japonais est une langue à accent mélodique (pitchaccent language). Néanmoins, les deux systèmes mettent en relief une syllabe en l'accentuant, ce qui permet ainsi une comparaison. En japonais, la syllabe accentuée est celle qui précède la chute du ton mélodique, lorsque cette chute existe. Dans le cas contraire, aucune syllabe n'est accentuée. La source dictionnairique utilisée pour noter le placement de la syllabe accentuée des items japonais est le Kenkyusha's New JapaneseEnglish Dictionary ${ }^{17}$ de Masuda Koh (1974). Cette version très complète présente l'avantage d'une écriture romanisée des mots japonais, qui sont en outre rangés par ordre alphabétique. Sur les 464 emprunts en anglais, 326 mots japonais sont recensés ${ }^{18}$ dans le Kenkyusha's. Quelques exemples de transcriptions de l'accentuation en japonais sont notés ci-dessous (les syllabes non accentuées sont notées en italique) :

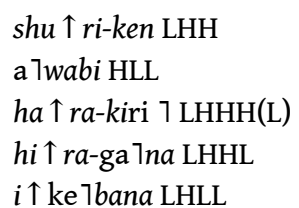

13 Le terme shuri-ken n'a pas de syllabe accentuée. En l'absence d'accent sur la première syllabe, l'une des particularités du système phonologique japonais est d'attribuer automatiquement un "ton» montant à la seconde syllabe, passant de L (pour Low) à $\mathrm{H}$ (pour High). Le ton reste ensuite "haut» (High) sur les syllabes suivantes si l'item ne reçoit pas d'accent. Le passage de L à $\mathrm{H}$ est indiqué par le symbole $\uparrow$. La présence de la 
syllabe accentuée est notée à l'aide du signe 7. La syllabe précédant la chute du ton mélodique est la syllabe accentuée en japonais, le ton passant ainsi de $\mathrm{H}$ à L. Les termes awabi et ikebana sont donc accentués sur la syllabe antépénultième, tandis que hara-kiri est accentué sur la finale et hira-gana sur la pénultième ${ }^{19}$.

Avant de procéder au travail de comparaison des transcriptions phonétiques des trois sources anglaises avec la transcription japonaise correspondante, la sous-partie suivante analyse l'accentuation des emprunts japonais en anglais britannique ${ }^{20}$.

\section{L'accentuation des emprunts japonais en anglais}

Le tableau 3 répertorie l'accentuation des emprunts dissyllabiques en anglais. Là encore, le caractère dissyllabique des 204 emprunts est déterminé à partir de la logique anglaise, tout comme le caractère trisyllabique (et plus) des emprunts du tableau 4.

Tableau 3. Accentuation des emprunts japonais dissyllabiques en anglais

\begin{tabular}{|c|c|c|c|}
\hline /01/ & /10/ & /01/-/10/ & total \\
\hline 9 & 187 & 8 & 204 \\
\hline $4,4 \%$ & $91,7 \%$ & $3,9 \%$ & $100 \%$ \\
\cline { 1 - 3 } go'bang & 'bonsai & banzai-ban'zai & \\
\cline { 1 - 3 } sen'sei & 'haiku & 'gaijin-gai'jin & \\
\cline { 1 - 3 } ty'coon & 'kana & 'Nikkei-Nik'kei & \\
\hline & 'manga & 'nisei-ni'sei & \\
\hline
\end{tabular}

Le tableau 4 répertorie les schémas accentuels des emprunts au japonais trisyllabiques et plus. Figurent dans ces cas particuliers, les emprunts pour lesquels une transcription phonétique est recensée dans l'OED, mais sans notation d'accent primaire. Ces termes inaccentués en anglais sont pourtant conservés car l'absence d'accentuation en anglais est peut-être la conséquence d'une absence d'accentuation en japonais. Mais il est plus probable qu'il s'agisse tout bonnement d'une erreur de transcription phonétique car chaque item est porteur d'un accent lexical en anglais. Sont également présents dans cette catégorie, les emprunts avec deux accents primaires. Or, les mots composés en anglais ne sont porteurs que d'un seul accent primaire. Le caractère compositionnel de l'emprunt semble avoir été nonobsté au profit d'une double accentuation primaire, caractéristique d'une séquence fortuite.

Tableau 4. Accentuation des emprunts japonais trisyllabiques (et plus) en anglais

\begin{tabular}{|c|c|c|c|c|c|c|}
\hline$/-1 /$ & $/-\mathbf{1 0} /$ & $/(-) \mathbf{1 0 0} /$ & $/(-) \mathbf{1 0 0 0} /$ & variation & cas particuliers & total \\
\hline 4 & 169 & 46 & 3 & 23 & 15 & 260 \\
\hline
\end{tabular}




\begin{tabular}{|c|c|c|c|c|c|c|}
\hline $1,5 \%$ & $65 \%$ & $17,7 \%$ & $1,2 \%$ & $8,8 \%$ & $5,8 \%$ & $100 \%$ \\
\hline & ad'zuki & 'anime & & ai'kido - 'aikido & hanami (/000)/ & \\
\hline jigo'tai & hara-'kiri & 'netsuke & 'Kamakura & 'sashimi-sa'shimi & hatamoto $(/ 0000) /$ & \\
\hline shuri'ken & kake'mono & 'samisen & 'sewamono & Su'doku - 'Sudoku & mo'kume 'gane & \\
\hline & Mi'kado & 'samurai & & 'bushido - bushi'do & 'shime-'waza & \\
\hline
\end{tabular}

17 Les emprunts japonais dissyllabiques en anglais sont massivement accentués en /10/ ( $91 \%$ contre $9 \%$ d'accentuation en $/ 01 /$ et de variation). Les résultats sont moins tranchés concernant les trisyllabes (et plus) : $65 \%$ sont accentués en /-10/ contre $18 \%$ en $/(-) 100 /$. Le phénomène variationnel est significatif puisque près de $9 \%$ des trisyllabes l'attestent. Enfin, $8 \%$ de schémas accentuels dits marginaux sont répertoriés.

Le facteur déterminant dans l'accentuation des emprunts japonais en anglais réside-t-il dans la conservation de l'accentuation de la langue d'origine? Le taux de correspondance très élevé entre l'italien et l'anglais se retrouve-t-il également entre le japonais et l'anglais? La partie suivante analyse le taux de correspondance entre les accentuations japonaise et anglaise.

\section{Étude de l'analogie accentuelle entre le japonais et l'anglais}

Le tableau 5 répertorie tous les résultats concernant la comparaison entre l'accentuation des termes japonais et des emprunts correspondants en anglais. La scission entre les emprunts dissyllabiques et trisyllabiques (et plus) est conservée, tout comme les cas pour lesquels aucune syllabe n'est accentuée en japonais. Logiquement, ces termes japonais sans accent excluent toute correspondance potentielle vis-à-vis de leurs homologues anglais.

Tableau 5. Étude de la correspondance accentuelle entre les termes japonais et les emprunts correspondants en anglais

\begin{tabular}{|c|c|c|c|c|c|}
\hline & & $\begin{array}{c}\boldsymbol{\emptyset}^{*} / \text { accent } \\
\text { JP / GB }\end{array}$ & $\begin{array}{c}\text { correspondance } \\
\text { JP / GB }\end{array}$ & $\begin{array}{c}\text { corr. partielle } \\
\text { JP / GB }\end{array}$ & $\begin{array}{c}\text { pas de corr. } \\
\text { JP / GB }\end{array}$ \\
\hline \multirow{5}{*}{ dissyll. } & chiffres & 70 & $48(58 \%)$ & $4(5 \%)$ & $31(37 \%)$ \\
\hline & $\%$ & $46 \%$ & \multicolumn{3}{|c|}{$54 \%$} \\
\hline & \multirow{3}{*}{ exemples } & bonsai / 'bonsai & budo / 'budo & mikan / 'mikan & bento / 'bento \\
\hline & & zazen / za'zen & ippon / 'ippon & nisei/'nisei & furo / 'furo \\
\hline & & kanji / 'kanji & judo / 'judo & $(+/ 01 / \mathrm{GB})$ & kata / 'kata \\
\hline \multirow{2}{*}{$\begin{array}{l}\text { trisyll. } \\
\text { (et plus) }\end{array}$} & chiffres & 104 & $15(22 \%)$ & $5(7 \%)$ & $49(71 \%)$ \\
\hline & $\%$ & $60 \%$ & \multicolumn{3}{|c|}{$40 \%$} \\
\hline
\end{tabular}




\begin{tabular}{|c|c|c|c|c|}
\hline \multirow{8}{*}{ exemples } & karaoke JP & gagaku JP & & hijiki JP \\
\hline & kara'oke GB & 'gagaku GB & & hi'jiki GB \\
\hline & kimono JP & hiragana JP & aikido JP & ikebana JP \\
\hline & ki'mono GB & hira'gana GB & ai'kido GB & ike'bana GB \\
\hline & samurai JP & kagura JP & $(+/ 100 / G B)$ & katana JP \\
\hline & 'samurai GB & 'kagura GB & wasabi JP & ka'tana GB \\
\hline & shuriken JP & marumage JP & 'wasabi GB & uchiwa JP \\
\hline & shuri'ken $G B$ & 'marumage $G B$ & $(+/ 010 / G B)$ & 'uchiwa GB \\
\hline
\end{tabular}

* Le symbole Ø signifie que le terme n'est pas accentué en japonais. Les syllabes accentuées en japonais dans le reste du tableau sont notées en caractères droits. japonais. En effet, $46 \%$ des mots étiquetés comme dissyllabiques en anglais ne sont pas accentués en japonais. Ce pourcentage s'accroît encore pour les mots plus longs car $60 \%$ des trisyllabes (et plus) ne sont pas accentués en japonais. L'étude d'une éventuelle correspondance accentuelle basée sur ces items n'est donc pas pertinente, car la langue anglaise requiert la présence au minimum d'une syllabe accentuée sur chaque unité lexicale.

21 Concernant les items accentués dans les deux langues, la tendance penche vers une absence de correspondance entre la position de la syllabe accentuée du terme japonais et celle de la syllabe accentuée en anglais. Pour les dissyllabes, il y a $58 \%$ de correspondance contre $37 \%$ de non-correspondance (et $5 \%$ de correspondance partielle). Pour les trisyllabes (et plus), le phénomène s'inverse car il n'y a que $22 \%$ de correspondance accentuelle contre $71 \%$ de non-correspondance (et $7 \%$ de correspondance partielle). Le pourcentage d'absence de correspondance serait encore accru si les termes japonais non accentués étaient inclus dans les calculs.

Aucun chiffre significatif ne laisse penser que le placement de l'accent / $1 /$ dans les emprunts japonais en anglais est directement déterminé par la position de la syllabe accentuée dans la langue d'origine. Lors du transfert lexical du japonais vers l'anglais, il y a donc un blocage de certains mécanismes phonologiques et en particulier des principes accentuels. La reproduction de l'accentuation d'origine semble bloquée par les différences fondamentales existant entre les mécanismes phonologiques de l'anglais et ceux du japonais. Comment dès lors justifier l'accentuation des emprunts japonais en anglais? Les tableaux de la partie précédente démontrent que le schéma accentuel privilégié est celui de la syllabe pénultième. Il semble exister en anglais un schéma accentuel en /(-)10/ qui serait propre aux mots étrangers et qui deviendrait justement un indicateur du caractère étranger du terme.

La partie suivante consacrée à l'accentuation des emprunts français en anglais englobe une nouvelle perspective sociolinguistique absente des analyses sur l'italien et le japonais. 


\section{L'accentuation des emprunts français en anglais}

\section{Constitution d'un corpus représentatif d'emprunts français}

24 La constitution du corpus est opérée à partir de terminaisons typiquement françaises car il serait utopique de vouloir rendre compte de l'accentuation de l'intégralité des emprunts français attestés en anglais contemporain. En effet, pour des raisons historiques, l'anglais a massivement emprunté au lexique français. Les terminaisons choisies sont les suivantes: <-aire>, <-euse>, <-eur>, <-eux>, <-C'C'e>21, <-que>, <-ier>, <ais $>,<-e a u>$ et $<-$ aux $>22$. Pour limiter la taille du corpus, une seule base de données est utilisée, à savoir l'EPD. Les vérifications d'ordre étymologique sont effectuées par l'intermédiaire de l'OED. Sont conservés les emprunts français au moins dissyllabiques, de même que les noms propres ${ }^{23}$. Enfin, les données de l'EPD relatives aux deux variétés d'anglais que sont l'anglais britannique et l'anglais américain sont incluses dans l'analyse car il s'avère que la variété d'anglais considérée a un impact non négligeable sur l'accentuation des emprunts français, à l'inverse de l'italien et du japonais. Le corpus d'emprunts français en anglais contemporain s'élève ainsi à 403 éléments ${ }^{24}$.

L'accentuation finale démarcative $d u$ français, permettant de délimiter les groupes intonatifs, est-elle reproduite en anglais ${ }^{25}$ par la présence d'un accent primaire sur la syllabe finale des emprunts ou bien les emprunts ont-ils subi un processus d'assimilation consistant en une rétraction de l'accent primaire sur la syllabe pénultième ou antépénultième en anglais?

\section{Résultats}

Le tableau suivant répertorie les schémas accentuels des 403 emprunts français en anglais britannique et en anglais américain notés dans l'EPD ${ }^{26}$ :

Tableau 6. Accentuation des emprunts français en anglais britannique et en anglais américain

\begin{tabular}{|c|c|c|c|c|}
\hline & $/-1 /$ & acc non finale & & /-1/ en variante \\
& & & total \\
\hline \multirow{2}{*}{ GB } & 237 & 105 & 61 & 403 \\
\cline { 2 - 5 } & $58,8 \%$ & $26,1 \%$ & $15,1 \%$ & $100 \%$ \\
\hline \multirow{2}{*}{ US } & 295 & 68 & 40 & 403 \\
\cline { 2 - 5 } & $73,2 \%$ & $16,9 \%$ & $9,9 \%$ & $100 \%$ \\
\hline
\end{tabular}

Le pourcentage d'emprunts français accentués sur la syllabe finale est plus important en anglais américain qu'en anglais britannique (73\% contre $58 \%$ ). Cependant, il s'avère que la catégorie de l'emprunt joue également un rôle dans son accentuation en anglais. Ainsi, le tableau 7 procède aux mêmes calculs que le tableau 6, tout en séparant les noms communs des noms propres. 
Tableau 7. Accentuation des noms communs et des noms propres d'origine française en anglais britannique et en anglais américain ${ }^{29}$

\begin{tabular}{|c|c|c|c|c|}
\hline \multirow{2}{*}{} & \multicolumn{2}{|c|}{ GB } & \multicolumn{2}{c|}{ US } \\
\cline { 2 - 5 } & $\mathrm{NC}^{30}$ & $\mathrm{NP}$ & $\mathrm{NC}$ & $\mathrm{NP}$ \\
\hline \multirow{2}{*}{$/-1 /$} & 190 & 45 & 220 & 73 \\
\cline { 2 - 5 } & $62,3 \%$ & $46,9 \%$ & $72,1 \%$ & $76 \%$ \\
\hline \multirow{2}{*}{ acc non finale } & 69 & 36 & 51 & 17 \\
\cline { 2 - 5 } & $22,6 \%$ & $37,5 \%$ & $16,7 \%$ & $17,7 \%$ \\
\hline /-1/ en variante & 46 & 15 & 34 & 6 \\
\cline { 2 - 5 } & $15,1 \%$ & $15,6 \%$ & $11,2 \%$ & $6,3 \%$ \\
\hline \multirow{2}{*}{ total } & 305 & 96 & 305 & 96 \\
\hline
\end{tabular}

La catégorie de l'emprunt ne modifie pas le pourcentage des accentuations finales et non finales en anglais américain. En effet, $76 \%$ des noms propres sont accentués sur la finale contre $72 \%$ des noms communs. En revanche, en anglais britannique, des différences importantes apparaissent en fonction de la catégorie de l'emprunt. Les noms communs conservent davantage l'accentuation finale caractéristique du français que les noms propres (62\% contre $46 \%$ ). Les noms propres affichent donc un fort pourcentage d'accentuation non finale en anglais britannique. Ce résultat peut paraître surprenant car la spécificité d'un nom propre est de renvoyer à une notion, à une figure identitaire. La prononciation fait partie intégrante de cette notion identitaire et nous aurions pu penser que cette catégorie d'items serait moins sujette au phénomène de rétraction accentuelle en anglais britannique et au contraire plus favorable à la conservation d'une prononciation «à la française ».

Une étude plus approfondie sur le taux de correspondance accentuelle entre les deux variétés d'anglais dans l'EPD montre qu'il est nettement inférieur aux résultats concernant l'italien ou le japonais, comme le montre le tableau suivant :

Tableau 8 : Correspondance accentuelle entre les variétés d'anglais britannique et américain dans I'EPD

\begin{tabular}{|c|c|c|}
\hline correspondance & non-correspondance & correspondance partielle \\
\hline $299 / 403$ & $37 / 403$ & $67 / 403$ \\
\hline $74,2 \%$ & $9,2 \%$ & $16,6 \%$ \\
\hline
\end{tabular}

Dans $16,6 \%$ des cas, il n'y a pas de correspondance accentuelle entre l'anglais britannique et l'anglais américain. L'accentuation des emprunts français en anglais intègre ainsi une dimension sociolinguistique absente des résultats concernant l'italien et le japonais. 


\section{Discussion}

31 français en anglais sont avancés dans l'article de Pierre Fournier (2013). L'existence d'une variation inter-variétale significative montre que l'accentuation finale française est mieux conservée en anglais américain qu'en anglais britannique où la tendance à la rétraction accentuelle est plus importante. La scission entre les noms propres et les noms communs accentue encore le phénomène car la rétraction accentuelle est encore plus forte pour les noms propres en anglais britannique. La proximité géographique entre la France et la Grande-Bretagne aurait-elle créé une rivalité socioculturelle? Les locuteurs britanniques souhaiteraient-ils minimiser l'influence du français, le meilleur exemple étant d'adopter le principe de la rétraction accentuelle afin de se démarquer d'une accentuation finale typique du français? Les emprunts français en anglais britannique seraient dès lors prononcés en adoptant des schémas accentuels plus conformes aux spécificités du système anglais. Ce phénomène, s'il s'avérait avoir un réel impact, ne serait pas conscient de la part des locuteurs natifs, mais plutôt sujet à des «effets de mode ». La prononciation « à la française » est peut-être moins en vogue actuellement en Grande-Bretagne qu'aux États-Unis. Ce premier argument difficilement vérifiable ne se base sur aucun réel fondement scientifique. Le second argument en revanche postule l'existence de morphologies déterminantes qui seraient différentes en anglais britannique et en anglais américain. Certaines terminaisons d'origine française formeraient des microsystèmes au sein du système britannique, pour lesquelles la logique de l'accentuation française subirait la pression de la logique germanique symbolisée par la rétraction accentuelle. Il apparaît en effet que les emprunts avec des terminaisons en <ier ${ }^{31}$ ou <-é> sont systématiquement accentués sur la syllabe pénultième en anglais britannique mais sur la syllabe finale en anglais américain.

\section{Conclusion}

Trois langues pour trois visions différentes de l'accentuation des emprunts en anglais. $\mathrm{Si}$ les emprunts à l'italien conservent et reproduisent fidèlement l'accentuation de la langue d'origine, les emprunts au japonais montrent le phénomène inverse avec un blocage total des mécanismes accentuels lors du transfert lexical et une accentuation déterminée à partir des spécificités de la phonologie anglaise. Cette conservation et cette reproduction de la syllabe accentuée ou au contraire son blocage et une réanalyse accentuelle basée sur le système anglais pourraient constituer les deux pôles selon lesquels l'accentuation des emprunts en anglais pourrait être étudiée. En effet, une typologie de l'accentuation des emprunts basée sur le degré de conservation des données accentuelles de la langue source pourrait permettre une classification des langues auxquelles l'anglais a emprunté avec, comme idée sous-jacente, la possibilité de l'existence d'un schéma accentuel distinctif des mots étrangers en anglais, à savoir /(-)10/, directement hérité des langues sources, intégré au système phonologique anglais, et automatiquement appliqué aux emprunts pour lesquels il ne peut y avoir de correspondance accentuelle avec la langue source correspondante. Les emprunts au français ont un statut particulier au sein de l'accentuation anglaise en raison de considérations historiques et du fait de l'impact de l'introduction du latin et du français sur la phonologie de l'anglais. La présence d'une 
variation inter-variétale et de sous-systèmes morphologiques sujets à ces variations font des emprunts français un exemple unique à la frontière entre conservation et réanalyse accentuelle. Enfin, la légitimité des formes institutionnalisées présentes dans les dictionnaires de prononciation doit être renforcée par des tests oraux sur des locuteurs natifs. Comment les emprunts intègrent-ils la langue anglaise? Par l'intermédiaire de l'écrit ou de l'oral? En effet, la filière d'intégration joue un rôle prépondérant dans la prononciation de l'emprunt dans la langue réceptrice.

\section{BIBLIOGRAPHIE}

Borrelli, Doris, 2002, Raddoppiamento Sintattico in Italian. A Synchronic and Diachronic Cross-dialectal Study, New York, Londres, Routledge.

Boulle, Jacques, 1984, "Suffixes latins et accentuation anglaise : aperçus sur une évolution historique ", dans M. Cling et J. Humbley (dir.), $2^{e}$ Colloque d'avril sur l'anglais oral, Villetaneuse, Université Paris-Nord, CELDA, diffusion APLV, p. 207-225.

Calabrese, Andrea et Wetzels, Léo, 2009, Loan Phonology, Amsterdam, John Benjamins.

Cannon, Garland, 1981, «Japanese Borrowings in English », American Speech, vol. 56, $\mathrm{n}^{\circ} 3$, p. 190-206.

Chadelat, Jean-Marc, 2000, Valeurs et fonctions des mots français en anglais à l'époque contemporaine, Paris, L'Harmattan.

Descloux, Élodie, Fournier, Pierre, Martin, Marjolaine et Vanhoutte, Sophie, 2011, « Les mots français en -eur (-euse) / -aire en anglais contemporain : emprunt et création lexicale ", dans M. Iliescu et al. (dir.), Actes du Colloque international. Les emprunts lexicaux au français dans les langues européennes, Cracovie, Universitaria, p. 145-162.

Eggs, Ekkehard et Mordellet-Roggenbuck, Isabelle, 1990, Phonétique et phonologie du français, Tübingen, Niemeyer.

Fournier, Pierre, à paraître b, « Transcription et formalisation de l'accentuation des mots italiens en anglais contemporain ", dans Transcrire, écrire, formaliser 2, Rennes, Presses Universitaires de Rennes, coll. « Travaux Linguistiques du CerLiCO ».

Fournier, Pierre, à paraître a, « Word Stress Assignment of Italian Loanwords in Contemporary English ».

Fournier, Pierre, 2013, «L'accentuation des emprunts français en anglais britannique et américain », Annales de l'Université de Craïova - Série langues et littératures romanes, vol. 17, nº 1 , p. 102-126.

Fournier, Pierre et Vanhoutte, Sophie, 2013, « Stress in Japanese Loanwords in English: Faithfulness or Adaptation? », communication effectuée à l'occasion de la International Conference on Phonetics and Phonology (ICPP2013), Tokyo.

Jones, Daniel, 2011, Cambridge English Pronouncing Dictionary (18 ${ }^{\text {th }}$ ed.), Cambridge, Cambridge University Press. 
Kenstowicz, Michael et Sohn, Hyang-Sook, 2001, « Accentual Adaptation in North Kyungsang Korean ", dans M. Kenstowicz (dir.), Ken Hale: A Life in Language, Cambridge, MA, The MIT Press, p. 239-270.

Koh, Masuda, 1974, Kenkyusha's New Japanese-English Dictionary, Tokyo, Kenkyusha, $4^{\mathrm{e}} \mathrm{ed}$

Labrune, Laurence, 2012, The Phonology of Japanese, Oxford, Oxford University Press.

Léon, Monique et Léon, Pierre, 2009, La Prononciation du français, Paris, Armand Colin.

Pinnavaia, Laura, 2001, The Italian Borrowings in the Oxford English Dictionary, Rome, Bulzoni.

Quinio, Julie, 2009, La Phonologie des emprunts français non anglicisés en anglais, Thèse de Doctorat, Université Paris IV.

Robert, Paul, et Arizzi, Augusto, 1999, Le Robert \& Signorelli. Dizionario Francese-Italiano/Italiano-

Francese, Paris, Dictionnaires Le Robert, Milano, Signorelli.

Sabatini, Francesco et Coletti, Vittorio, 2005, Dizionario della Lingua Italiana, Milan, Rizzoli-

Larousse.

Simpson, John et Weiner, Edmund, 1989, Oxford English Dictionary. Online Edition, Oxford, Clarendon Press.

Svensson, Ann-Marie, 2001, « Germanic vs French Stress in Disyllabic Loan Words in English », Gothenburg Studies in English, n 81, p. 123-129.

Thornton, Anna M., Iacobini, Claudio et Burani, Cristina, 1997, BDVDB Una base di dati sul vocabolario di base della lingua italiana, Rome, Bulzoni.

Vance, Timothy J., 2008, The Sounds of Japanese, Cambridge, Cambridge University Press.

Wells, John C., 2008, Longman Pronunciation Dictionary, Londres, Longman, $3^{\mathrm{e}}$ éd.

\section{NOTES}

1. Et en particulier la présence d'un agrégat consonantique en position préfinale.

2. L'accentuation en italien est principalement pénultième. Si l'anglais conserve l'accentuation italienne originelle dans les emprunts, l'accentuation des emprunts en anglais sera pénultième ou à défaut antépénultième, car la majorité des mots longs en anglais est accentuée sur la syllabe antépénultième. Les emprunts dissyllabiques ne sont pas conservés car l'accentuation finale étant rare en italien, les schémas accentuels privilégiés pour observer le taux de correspondance sont les schémas sur la pénultième ou l'antépénultième (ce dernier excluant de fait les dissyllabes).

3. En effet, les emprunts avec un agrégat consonantique préfinal peuvent également relever de la règle du poids syllabique, productive en anglais. En d'autres termes, si un emprunt italien en $<\mathrm{VC}$ ${ }_{2} \mathrm{~V} \#>$ est accentué sur la syllabe pénultième à la fois dans la langue source et dans la langue réceptrice, il est impossible de savoir si la position de l'accent primaire de l'emprunt en anglais est déterminée par la conservation de l'accentuation italienne ou au contraire par l'influence de l'agrégat consonantique en position préfinale retenant l'accent en anglais. Il est donc préférable de ne pas conserver les emprunts présentant cette structure.

4. Les cas suivants présentent une structure syllabique finale modifiée ne permettant pas de comparer la position de la syllabe accentuée dans les deux langues : teleferica (italien) $\rightarrow$ teleferic (anglais), donzello $\rightarrow$ donzel, etc. De même, les emprunts italiens avec un <e\#> final non prononcé en anglais ne sont pas pertinents car l'italien prononce le $<\mathrm{e} \#>$, ce qui modifie encore une fois la structure syllabique de l'emprunt. 
5. Voir Doris Borrelli (2002) ; Anna M. Thornton, Claudio Iacobini et Cristina Burani (1997).

6. Le transfert provoque un processus de resyllabification en anglais qui se traduit par un traitement distinct: le système syllabique anglais considère que les séquences monosyllabiques $<-i+\mathrm{V}>$ sous accent en italien sont dissyllabiques en anglais : a'rioso [jo] (italien) $\rightarrow$ ari'oso (anglais).

7. La variété d'anglais retenue pour cette analyse est celle de l'anglais britannique. En effet, le pourcentage de variation entre l'anglais britannique et américain étant extrêmement faible concernant l'accentuation des emprunts à l'italien, les données par défaut sont celles de la variété britannique.

8. Le terme à gauche de la flèche représente le terme italien, et celui à droite, l'emprunt correspondant en anglais.

9. Les cas de variation en genre et en nombre de l'italien à l'anglais n'étant pas déterminants dans l'assignation de l'accent / $1 /$, ils sont donc incorporés à l'étude.

10. Ces 13 termes ne sont pas inclus dans les calculs du tableau précédent car la détermination du schéma accentuel pose problème. Le problème provient $d u$ statut des séquences $<-i+V>$ en italien et en anglais (voir note 6). Les séquences en $<-i+V>$ sont considérées comme dissyllabiques en anglais et les emprunts relèvent dès lors d'un schéma accentuel en /(-)100/ alors que la tradition phonologique italienne attribue un statut monosyllabique à ces mêmes séquences. Le but de cet article ne consistant pas à traiter du statut de la syllabe, la correspondance accentuelle fait l'objet d'une évaluation basée sur une simple observation de la position de l'accent.

11. L'exception de balcony semble démontrer que plus un emprunt est anglicisé et moins il subit l'influence accentuelle de la langue source.

12. Le chiffre (3) signifie que le schéma accentuel associé est attesté dans les trois sources dictionnairiques.

13. La variation peut être de nature inter-dictionnairique ou intra-dictionnairique. La nature de la variation n'étant pas déterminante, les deux types sont englobés sans distinction au sein des données.

14. Les traductions littérales les plus représentatives sont celles de black belt ou de martial art.

15. Les emprunts qualifiés d'hybrides sont en général des mots composés d'un emprunt japonais, associé à une traduction anglaise (exemples : happi-coat, Mikimoto pearl).

16. Ces emprunts sont qualifiés de monosyllabes en vertu des spécificités syllabiques de l'anglais. En japonais, en revanche, le statut des nasales finales peut conférer un statut dissyllabique à certains de ces items.

17. La variété japonaise de référence est la langue parlée dans la région de Tokyo, mais il existe encore à l'heure actuelle des débats autour de la variété standard de référence.

18. Il en existe en réalité sans doute davantage, mais la transcription des symboles japonais en écriture romanisée a vraisemblablement provoqué certaines modifications graphiques rendant la recherche des items parfois difficile.

19. Pour un traitement plus détaillé de l'accentuation en japonais, voir Laurence Labrune (2012) et Timothy J. Vance (2008).

20. Comme pour l'italien, le recours aux données concernant l'anglais américain n'est pas nécessaire, le taux de variation entre les deux variétés n'étant pas suffisamment significatif.

21. $<-C^{\prime} C^{\prime} e>$ fait référence à des géminées consonantiques (séquence de deux consonnes identiques) suivies d'un $<\mathrm{e}>$ final.

22. Les terminaisons finales retenues ont un effet normalisateur qui oriente l'étude vers une harmonisation de l'accentuation finale par rapport à des emprunts sans terminaison / suffixe remarquable.

23. À l'exception de l'italien et du japonais où la quasi-totalité des emprunts sont des noms communs, l'anglais a beaucoup emprunté de noms propres au français. Ils sont conservés car, audelà des noms propres toponymiques ou faisant référence à des personnages historiques français, sont également attestées beaucoup de marques françaises dans le lexique anglais, ces items 
fonctionnant presque comme des termes du vocabulaire courant. De plus, les noms propres français semblent régis par les mêmes principes accentuels que les noms communs, ce qui nous conduit à les inclure dans l'analyse.

24. Pour une description précise de toutes les étapes de constitution du corpus, ainsi que quelques perspectives historiques concernant les emprunts français en anglais, mais également quelques données phonologiques et l'impact que l'introduction des emprunts français a eu sur la phonologie de l'anglais, voir Jacques Boulle (1984), Élodie Descloux et al. (2011) ; Pierre Fournier (2013). L'intégralité du corpus des emprunts français utilisé dans cette étude est également disponible en annexe de l'article de Pierre Fournier (2013).

25. Pour une description plus détaillée de l'accentuation du français, voir Monique Léon et Pierre Léon (2009), Ekkehard Eggs et Isabelle Mordellet-Roggenbuck (1990). En outre, l'accentuation des emprunts français en anglais a déjà fait l'objet de travaux parmi lesquels peuvent figurer : JeanMarc Chadelat (2000) et Julie Quinio (2009). Cette dernière postule qu'il existe une différence de traitement en anglais entre les emprunts qui sont dits assimilés et ceux qui sont non assimilés. L'accentuation et la prononciation de ces emprunts en anglais constituent donc un critère de classification.

26. Tous les tableaux concernant les emprunts français sont extraits de Pierre Fournier (2013).

27. Par accentuation non finale, il faut comprendre les emprunts accentués principalement sur la syllabe pénultième $(/(-) 10 /)$ ou antépénultième $(/(-) 100 /)$. Il est légitime de regrouper les accentuations non finales dans une seule et même catégorie car le but de l'analyse est de déterminer si l'anglais reproduit ou non l'accentuation française finale.

28. L'accentuation finale figure en variante principale ou en variante secondaire dans les données de l'EPD.

29. Deux emprunts ont une double utilisation de nom propre et de nom commun (bastille / Bastille et nouvelle vague / Nouvelle vague). Ils sont donc retirés des calculs figurant dans le tableau. Au total, sur 401 emprunts français, nous identifions 305 noms communs et 96 noms propres.

30. « NC » pour nom commun et « NP » pour nom propre.

31. La terminaison <-ier> bénéficie d'un statut particulier en anglais en raison de l'interférence avec la famille des mots en $\{\mathrm{i}, \mathrm{e}, \mathrm{u}\}+\mathrm{V}\left(\mathrm{C}_{0}(\mathrm{e})\right)$ avec deux traitements distincts : terminaison traitée comme dissyllabique ou comme monosyllabique.

\section{RÉSUMÉS}

Cet article tente de rendre compte de l'accentuation des emprunts en anglais contemporain, et en particulier de la position de l'accent primaire (noté /1/). Le recours aux trois langues sources que sont l'italien, le japonais et le français, langues auxquelles l'anglais a historiquement beaucoup emprunté, permet de mettre en évidence trois aspects des transferts de données phonologiques lors du phénomène d'emprunt et d'envisager une typologie des emprunts en anglais basée sur la relation langue source-langue réceptrice, à la croisée entre conservation / reproduction et blocage / assimilation.

This article investigates the stress patterns of loanwords in contemporary English, and in particular the primary stress location $(/ 1 /)$. Using three source languages (Italian, Japanese and French), it turns out that three distinct aspects of phonological transfers are highlighted and that the creation of a typology of loanwords in English, which would be based on the connection 
between the source language and the target language is made possible with the parameters of faithfulness/replication and assimilation/adaptation.

INDEX

Mots-clés : accentuation, emprunts, anglais contemporain, contacts entre langues, conservation, adaptation

Keywords : stress, loanwords, contemporary English, loan phonology, faithfulness, adaptation

\section{AUTEUR}

PIERRE FOURNIER

Université Paris 13, Sorbonne Paris Cité, Pléiade (EA 7338) 